\title{
Advances in the diagnosis and treatment of achalasia of the cardia: A review
}

\author{
Baozhen Zhang, Yidan Wang, Ye Liao, Jingjing Zhang, Yufan Wu, Tingyue Xiao, \\ Yue Zhang, Yiwen Bao, Hongyu Qiu, Siyu Sun, Jintao Guo \\ Department of Gastroenterology, ShengJing Hospital of China Medical University, Shenyang, \\ Liaoning Province, China
}

\section{ABSTRACT}

Idiopathic achalasia is an esophageal motor disorder characterized by the loss of the lower esophageal sphincter ganglion, resulting in impaired lower esophageal relaxation and absence of esophageal peristalsis. Patients commonly present with progressive dysphagia accompanied by reflux, heartburn, retrosternal pain, and severe weight loss. Diagnosis is primarily based on the patient's chief complaints, barium esophagography, and the most recent high-resolution manometry. Endoscopic assessment and endoscopic ultrasonography also have significant value with regard to the exclusion of esophageal anatomical lesions, neoplastic diseases, and pseudoachalasia. However, as most patients with achalasia demonstrate a gradual onset, early diagnosis is difficult. Currently, treatment of idiopathic achalasia, including pneumatic dilation, stent placement, and surgical myotomy, is aimed at reducing lower esophageal sphincter pressure and relieving the symptoms of dysphagia. Peroral endoscopic myotomy has gradually become the mainstream treatment because it causes less trauma and has a rapid recovery rate. This article reviews the main methods of diagnosis and treatment of achalasia, with an emphasis on the potential of peroral endoscopic myotomy and the advancements of immunotherapy for achalasia.

Key words: achalasia, esophageal manometry, endoscopy, peroral endoscopic myotomy

Address for Correspondence: Prof. Siyu Sun and Dr. Jintao Guo, Department of Gastroenterology, ShengJing Hospital of China Medical University, No. 36, Sanhao Street, Shenyang 110004, Liaoning Province, China. E-mails: sun-siyu@163.com; guojt@sj-hospital.org

\begin{tabular}{|l|}
\hline Access this article online \\
\hline Website: \\
www.intern-med.com \\
\hline DOI: \\
10.2478/jtim-2021-0009 \\
\hline Quick Response Code: \\
\hline
\end{tabular}

\section{INTRODUCTION}

Achalasia (AC) is a major primary esophageal motor disorder with a reported incidence of approximately $1.63 / 100,000$ of population. ${ }^{[1]}$ However, recent studies have shown that the actual incidence is far higher than that previously reported. ${ }^{[2-3]}$ Presently, there is a lack of epidemiological investigations involving AC in China. AC may occur at any age, but a relatively high incidence has been found within the 20- to 50-year age group.

AC is primarily characterized by the absence of normal esophageal peristalsis and a defective relaxation function in the lower esophageal sphincter (LES) upon deglutition. For most patients with AC, onset is gradual, which makes early diagnosis difficult. The main clinical manifestations of AC include progressive dysphagia, reflux, vomiting, chest pain, respiratory symptoms (nocturnal cough, recurrent aspiration, and pneumonia), and weight loss..$^{[4-5]}$ At present, the etiology and pathogenesis of AC have not been fully elucidated. The disease is often thought to be either autoimmune, viral immune, or neurodegenerative. Many contemporary hypotheses have suggested that inflammation in the myenteric plexus of the LES may be caused by multifactorial mechanisms (psychiatric factors, viral infection, and genetic factors), which may trigger an autoimmune response in genetically susceptible individuals. This leads to the destruction of the inhibitory ganglion cells in the LES by autoimmune-mediated inflammation, resulting in the dysfunction of LES relaxation, and ultimately, the development of AC. Therefore, the autoimmune-mediated inflammatory response may play a pivotal role in the 
pathogenesis of AC. ${ }^{[6]}$ Current examination methods for AC include barium esophagography, esophageal manometry, endoscopy, and endoscopic ultrasonography (EUS). Treatment methods, including pharmacological, endoscopic, and surgical therapies, are mainly aimed at reducing LES pressure and the relief of symptoms.

In this paper, we present a review of the current diagnostic and treatment methods for AC.

\section{DIAGNOSIS OF ACHALASIA}

\section{Clinical manifestations of achalasia}

The primary clinical manifestation of AC is dysphagia, with disease progression occurring in the majority of patients. Early symptoms of AC are often indiscernible owing to the unapparent etiology and gradual progression. As the major symptoms experienced by certain patients include acid regurgitation, heartburn, and costal cartilage or retrosternal pain, AC is often mistaken for gastroesophageal reflux disease (GERD) during the early stages, thereby leading to a delay in accurate diagnosis and treatment. ${ }^{[7-8]}$ With the continued food intake by patients, the severity of reflux worsens as the accumulation of food residue within the esophagus increases. In severe cases, reflux occurs even during food intake or sleep, and the aspiration of refluxed gastric content can result in respiratory diseases such as aspiration pneumonia and lung abscesses. The risk of esophageal cancer in AC patients is also increased because of esophageal bruising and inflammatory irritation caused by long-term stasis of food in the esophagus. ${ }^{[0-10]}$ Patients with long-standing AC also experience significant weight loss because of the increased severity of both dysphagia and vomiting.

At this point in time, examinations such as EUS and computed tomography (CT) should be performed to differentiate between AC and pseudoachalasia (pseudo AC), which is caused by malignancies of the esophagogastric junction (EGJ), such as carcinoma of the cardia.

\section{Diagnostic methods for achalasia}

\section{Barium esophagography}

Currently, barium esophagography is one of the most convenient and widely used diagnostic methods for the diagnosis of AC in clinical practice. During the early stages of AC, esophageal dilation is insignificant, which allows the smooth entry of barium into the stomach. ${ }^{[1]}$ However, with disease progression, mild esophageal dilation occurs, resulting in a bird-beak narrowing of the EGJ. Consequently, an air-fluid level may be observed along with the absence of a gastric air bubble. In advanced cases, esophagography may reveal a large food and saliva stasis and a sigmoid-like appearance in the esophagus. Barium esophagography has several advantages: it is simple and low cost with a high level of patient adherence and high diagnostic accuracy. Furthermore, it enables clinicians to rule out anatomical abnormalities in the esophagus (e.g., diverticula, constriction, hiatal hernia, etc.) and assess the degree of esophageal dilation. Timed barium esophagogram is a novel, simple, noninvasive, and widely applicable barium swallowing technique, which involves image capture 1,2 , and 5 minutes after the ingestion of a fixed quantity of barium, with the purpose of image acquisition at the 2 -minute time point being the assessment of interim esophageal emptying. This method can be used to assess esophageal emptying in AC patients, and it enables objective post-treatment assessment for prediction treatment success. ${ }^{[12]}$

\section{Esophageal manometry}

Regardless of the barium esophagography and endoscopy findings, esophageal manometry is currently regarded as the gold standard for the diagnosis of $A C,{ }^{[13]}$ with lowcompliance infusion being the traditional method used in manometric evaluations. Manometry typically shows an aperistaltic esophageal body and, in some cases, elevated intraesophageal pressure due to food and saliva stasis and incomplete relaxation of the LES upon deglutition (residual pressure $>10 \mathrm{mmHg}$ ). ${ }^{[14]}$ The emergence of a novel manometric technique known as high-resolution manometry (HRM) has enabled the observation of movements in various sections of the esophagus with greater clarity, allowing for early diagnosis and classification of primary esophageal motor disorders, prediction of treatment response in patients, and enhancement of diagnostic reproducibility and sensitivity. ${ }^{[15-17]}$ Compared with traditional manometric methods, HRM enhances the sensitivity of AC diagnosis remarkably and provides an accurate assessment of parameters such as the degree of LES relaxation and amplitude and rate of esophageal contractions. According to the Chicago classification of AC, which involves the use of the average integrated relaxation pressure measured by HRM combined with dysphagia or esophageal spasms for the diagnosis of AC, the disease can be classified into the following three clinical subtypes: (1) Type I AC (classic): no pressure waves recorded in the distal esophagus; (2) Type II AC (with compression): panesophageal pressurizations; and (3) Type III AC (spastic): compartmentalized contractions in the distal esophagus or spastic contractions $>20 \% \cdot{ }^{[18]}$ It is important to note that the effects of AC treatment outcomes are dependent on the Chicago classification. Current studies have unanimously reported that the best and worst treatment effects have been achieved in Types II and III AC patients, respectively. ${ }^{[19-20]}$ Therefore, classification on the basis of HRM enables the prediction of treatment response in $\mathrm{AC}$ patients and facilitates the formulation of optimal individualized treatment regimens for patients. 


\section{Endoscopy}

Endoscopy is an essential method in the diagnosis of AC. All patients with suspected AC should undergo endoscopic examination to rule out pseudo AC, as well as esophageal anatomical lesions and neoplastic diseases, which manifest similar clinical symptoms and manometric results. Most cases of pseudo AC are caused by tumors of the EGJ. For elderly patients or patients with rapidly progressing dysphagia and significant weight loss, the possibility of pseudo AC should be considered. The EGJ region should be scrutinized during endoscopic examination, and EUS or CT should be subsequently performed in such cases to obtain a confirmed diagnosis. Studies have shown that both endoscopy and barium esophagography are less sensitive than manometry and only identify about half (or even less) of patients with early-stage AC. ${ }^{[21-22]}$ During the progression of AC, endoscopy may reveal bending and elongation of the esophageal body, significant esophageal dilation, a large amount of retained food within the esophageal lumen, tight closure of the cardia, and increased resistance at the EGJ. ${ }^{[23]}$ During endoscopic examination, the cardia and fundus should be scrutinized, and EUS and/or CT should be performed as necessary to aid with the diagnostic process. Based on morphological changes in the lower esophagus and cardia observed under endoscopic vision, Chinese researchers Li and Linghu proposed the Ling classification system for preoperative assessment of the feasibility of performing peroral endoscopic myotomy (POEM) in AC patients. ${ }^{[2]}$ Under this classification system, AC is classified as follows: (1) Type I: smooth esophageal lumen with absence of multi-ring, crescent-like, and diverticulum structures; (2) Type II: presence of a multi-ring or crescentlike structure and absence of a diverticulum structure; and (3) Type III: presence of a diverticulum structure, with Types II and III AC further divided into three subtypes each for the assessment of the feasibility of POEM in AC treatment. Results of the study indicated that the surgical success rate was highest in patients with Ling Type I AC.

\section{Endoscopic ultrasonography}

EUS is widely used in the diagnosis and treatment of digestive tract diseases, as it allows for a clear view of the layered structure and thickness of the esophageal wall. ${ }^{[25-31]}$ This has unique diagnostic value for submucosal lesions, as it enables the identification of pseudo AC or secondary AC caused by venous tumors, neurofibroma, cicatricial stenosis, systemic sclerosis, sarcoidosis, or malignant neoplasms, reducing the likelihood of misdiagnosis and mistreatment. EUS can also be performed before and after POEM to determine the required degree of muscularis propria division. Preoperative EUS enables measurement of the esophageal muscle layer thickness, in particular the inner circular layer, to predict the degree of complexity of the POEM procedure. Furthermore, postoperative
EUS enables the assessment of treatment effectiveness. ${ }^{[32]}$ According to Zhong et al. ${ }^{[33]}$ the ratio of esophageal annular muscle layer provided by EUS is expected to become one of the diagnostic criteria for AC. Further research has shown that EUS provides guidance for preoperative, intraoperative, and postoperative assessments of the treatment effects of POEM. For instance, the indication of uneven thicknesses in the muscularis propria of a patient by EUS may prompt the creation of the submucosal tunnel and the division of muscularis propria at the point of maximum thickness identified by the ultrasonographic probe. Such individualized POEM procedures consequently enable a reduction in postoperative complications and enhance surgical safety. Therefore, EUS not only provides information regarding identification and diagnosis of $\mathrm{AC}$, but also facilitates preoperative and postoperative assessments of the treatment effects of POEM. ${ }^{[34]}$

\section{Other diagnostic methods}

Esophageal emptying tests, such as the radionuclide esophageal transit study and the barium esophageal transit study, are primarily used to assess the transit and emptying of food in the esophagus, facilitating evaluation of the esophageal emptying function and assessment of treatment effects. Rapid radionuclide or barium clearance can be achieved in an individual with normal esophageal emptying function. By contrast, there will be a significant delay in radionuclide or barium clearance in patients with AC. Other diagnostic methods include abdominal ultrasonography and the administration of acetyl-betamethylcholine chloride; however, these methods are not widely used in clinical practice.

\section{TREATMENT OF ACHALASIA}

\section{Pharmacological therapy}

The aim of pharmacological therapy is to alleviate esophageal obstruction through LES relaxation, thereby providing temporary symptomatic relief. However, as pharmacological therapy is incapable of eliminating the underlying disease process, it often serves as the initial therapy. The two most commonly used medications in the pharmacological treatment of AC are calcium channel blockers and long-acting nitrates. ${ }^{[13,23]}$ Calcium channel blockers can be used in AC treatment, as they inhibit calcium ion transmembrane transport and calcium release, which enables the relaxation of the esophageal smooth muscle and reduction of LES pressure and resistance during deglutition. Long-acting nitrates act to reduce LES pressure through the release of nitric oxide (NO) or NO-related molecules, thereby relieving clinical symptoms. However, as these medications can cause side-effects such as dizziness, headache, lower extremity edema, and hypotension, as well as the development of 
tolerance after long-term use, pharmacological therapy is rarely used in current clinical practice; instead, it is usually used as an adjuvant therapy for early-stage AC, elderly high-risk individuals, or patients who refuse to undergo other therapies.

\section{Endoscopic treatment}

Endoscopic botulinum toxin injection (EBTI)

Botulinum toxin (Botox) is a paralytic toxin that causes paralysis by binding with presynaptic cholinergic terminals, inhibiting the release of acetylcholine from presynaptic neurons at neuromuscular junctions. This causes a loss of innervation in the motor endplate, thereby resulting in smooth muscle relaxation. In recent years, Botox has been widely used in the treatment of various muscle spasticity disorders and has achieved good treatment outcomes. The adoption of endoscopic botulinum toxin injection (EBTI) for the treatment of AC has several advantages, including reduced trauma, reduced discomfort, accurate positioning, good safety and effectiveness, ease of surgery, and low costs. However, EBTI can result in minor complications such as transient chest pain, reflux symptoms and, in rare cases, mediastinitis and allergic reactions. Repeated EBTI may also result in an inflammatory response and fibrosis in the mucosa and muscle layers, thereby increasing the risk and difficulty of subsequent surgery. ${ }^{[35]}$ EBTI has good, short-term effects. However, the effects of Botox fade gradually with time, leading to a relapse in more than $60 \%$ of patients 1 year after treatment. Approximately $50 \%$ of patients require further injections $6-24$ months after the first treatment, or additional treatments by pneumatic dilation and myotomy. ${ }^{[36]}$ Therefore, EBTI is more appropriate for frail and elderly individuals, patients with severe comorbidities, or those who refuse to undergo other therapies. ${ }^{[37]}$

\section{Endoscopic pneumatic dilation (EPD)}

Prior to the advent of POEM, pneumatic dilation (PD) was the treatment of choice for non-surgical treatment of AC. Endoscopic pneumatic dilation (EPD) is a physical therapy method that involves the use of external force to dilate the LES, causing certain muscle fibers of the LES to rupture, leading to the reduction of LES pressure. This facilitates the passage of food through the lower esophagus, thereby alleviating AC symptoms. EPD boasts several advantages, including ease of surgery, low cost, and notable treatment effects. Studies have shown that PD can be $60-85 \%$ effective, with the effects of a single PD lasting for more than 5 years in $75 \%$ of patients. ${ }^{[38]}$ In clinical practice, the most commonly used dilator is the Micro-invasive Rigiflex Balloon system (Boston Scientific Corp., Boston, MA, USA) with 3 diameter sizes $(3.0 \mathrm{~cm}, 3.5 \mathrm{~cm}$, and $4.0 \mathrm{~cm})$, which can be used for single or sequential dilation. Previous research has suggested that the $3.0 \mathrm{~cm}$ balloon for the first dilation should be used for most patients, and results have indicated that sequential PD is more effective than single PD using the $3.0 \mathrm{~cm}$ balloon. ${ }^{[39]}$

For patients who choose to undergo EPD, surgical treatment preparations are required, as the most serious complication associated with PD is esophageal perforation, which occurs in approximately $2 \%(0-16 \%)$ of cases and requires surgery in $50 \%$ of cases. Most cases of perforation occur during the first dilation because of the difficulty involved in placing the balloon in an appropriate position. In a study by Vanuytsel et al. ${ }^{[40]}$ it was found that the most important risk factors for esophageal perforation were age $>65$ years and the diameter of the balloon used during the first dilation $(\geq 3.5 \mathrm{~cm})$. Non-surgical treatment methods such as parenteral nutrition, anti-inflammatory treatment, and endoscopic clip closure can be adopted for most perforations, whereas surgical repair through thoracotomy is required for a few cases in which perforations are difficult to treat, resulting in severe symptoms, or are accompanied by mediastinal effusion and infection. Another major complication associated with EPD is GERD, which is usually alleviated by the administration of standard antacid therapy. In general, as EPD provides good long-term effects and is relatively safe, it is regarded as an effective non-surgical therapy for AC.

\section{Endoscopic stent placement (ESP)}

Endoscopic stent placement (ESP) for the treatment of $\mathrm{AC}$ involves the placement of a specially fabricated stent at the constricted region of the cardia. By virtue of its good tissue biocompatibility and shape-memory properties, the stent subsequently expands at body temperature to the maximum predicted diameter. This leads to the tearing of the muscularis propria and destruction of the LES structure and function, resulting in alleviation of dysphagia symptoms. Stent placement may be permanent or temporary. Although permanent ESP enables a more significant alleviation of short-term clinical symptoms, severe complications such as serious and frequent GERD and recurrent constriction have been observed over a longterm follow-up. ${ }^{[41]}$ For temporary ESP, inconsistencies exist in the clinical effects reported by different researchers. For instance, Li et al. ${ }^{[42]}$ found that the clinical effectiveness of metallic stents with a diameter of $30 \mathrm{~mm}$ was superior to those of metallic stents with a diameter of $25 \mathrm{~mm}$ or PD. However, the results obtained by Zhao et al. ${ }^{[43]}$ showed that stents with a larger diameter resulted in an increase in postoperative chest pain and reflux symptoms in AC patients. The key complications of ESP are stent dislodgement and migration, which may result in esophageal bleeding, ulcers, or perforations. A study by Rieder et al..$^{[4]}$ has suggested that endoscopic suture fixation of esophageal stents appears to be effective in preventing 
early migration of stents placed in difficult locations, such as the gastric/gastroesophageal junction (GEJ). With the emergence of other treatment techniques for AC, ESP is now less commonly adopted in clinical practice.

\section{Peroral endoscopic myotomy (POEM)}

Since the first series of successful POEM procedures in AC patients was reported in 2010 by Inoue et al., POEM has become an increasingly recognized form of therapy for AC. ${ }^{[45]}$ Relevant research has recommended the adoption of POEM as first-line therapy for AC owing to its minimal invasiveness, high feasibility, and good safety and effectiveness profiles. ${ }^{[4-47]}$ The POEM procedure involves the following: mucosal incision, creation of a submucosal tunnel, dissection of muscle fibers, and tunnel closure. As surgical maneuvers are performed on the esophageal mucosa under the endoscope, mediastinal contamination is extremely rare; therefore, resection of auxiliary anatomical structures of the LES, such as the phrenoesophageal ligament, is not required. Compared with Heller myotomy, which is another surgical procedure, POEM offers significant advantages such as reduced trauma, low cost, few complications, short hospitalization, and an absence of incisions on the body surface while also providing remarkable treatment effects. Therefore, POEM has gained increasing attention from gastroenterologists in recent years. Major limitations of the POEM technique include the difficulty of the procedure, which places a high demand on the technical expertise of endoscopic physicians and the long surgical time because of the creation of the submucosal tunnel, which increases the risk of insufflation-related complications such as subcutaneous emphysema. Short-term postoperative complications of POEM include insufflation-related adverse events (subcutaneous emphysema, mediastinal emphysema, pneumoperitoneum, and pneumothorax), hemorrhage, pyrexia, pleural effusion, perforation, and mediastinal and pulmonary infections. As the POEM technique does not include an anti-reflux procedure, the primary long-term complication in patients who undergo POEM is GERD. Consequently, AC patients without reflux symptoms may potentially develop pathological reflux after POEM. ${ }^{[23,48]}$ However, Rösch et al. ${ }^{[49]}$ asserted that it is possible that reflux ultimately occurs when any form of myotomy is performed, with the onset being earlier for POEM. Although POEM results in a high rate of postoperative reflux, it also provides superior treatment effects within 1 year of treatment. Therefore, the POEM technique and the consequent postoperative reflux should be viewed critically.

In recent years, modified POEM procedures such as transverse-incision POEM (T-POEM) have been developed by researchers, with relevant studies indicating superior treatment effects and safety than those achieved with the traditional POEM technique. ${ }^{[50]}$ Linghu first proposed modified POEM techniques that reduce the relevant surgical risks and allow for the expansion of indications for the traditional POEM procedure. These are termed short-tunnel POEM and POEM with simultaneous submucosal and muscle dissection (POEM-SSMD) and are used for patients with Ling Type IIc AC or severe adhesions between the mucosal and muscular layers after myotomy. ${ }^{[1-52]}$ Another modified POEM procedure, known as the Liu-POEM technique, has been proposed by Liu ${ }^{[53]}$ In contrast to traditional POEM, which involves the four-step process of mucosal incision, submucosal tunnel creation, circular muscle dissection, and tunnel closure, Liu-POEM is able to achieve myotomy and submucosal tunnel creation in a single step. In addition to the advantages of traditional POEM such as reduced trauma, few complications, and good treatment effects, Liu-POEM has the added benefits of a shorter surgical time and reduced postoperative complications such as insufflation-related adverse events and hemorrhage. However, as the technique is relatively new, further studies are required for the validation of its clinical effects and long-term complications.

\section{Surgical therapy}

Laparoscopic Heller myotomy (LHM)

In 1913, German surgeon Ernst Heller first described the surgical treatment of AC that involved cutting the LES via thoracotomy and reported remarkable treatment effects. The procedure, currently known as Heller myotomy (HM), can be performed using either a thoracoscopic or laparoscopic approach. The results of a study by Campos et al. ${ }^{[54]}$ indicated that better symptom relief was achieved with laparoscopic HM than with thoracoscopic HM. This may be attributed to the fact that the laparoscope offers a clear view of the anatomical structures of the esophagus and enables nimble maneuvers on the esophagus and meticulous dissection of the muscularis propria. With the traditional HM technique, the anti-reflux barrier function of the LES is damaged owing to significant surgical trauma, which leads to the frequent occurrence of postoperative reflux. Therefore, HM is usually performed in combination with fundoplication for the prevention of postoperative reflux in patients. Dor fundoplication is generally the procedure of choice for the treatment of reflux. Although Toupet and Nissen fundoplications can also prevent abnormal postoperative reflux effectively, the recurrence rate of dysphagia is significantly higher among patients who undergo Nissen fundoplication. ${ }^{[55]}$ Therefore, laparoscopic Heller myotomy (LHM) combined with Dor fundoplication (LHM + Dor) is widely recognized as the procedure of choice for the surgical treatment of AC. 


\section{Esophagectomy}

Despite effective treatment modalities such as PD and myotomy, approximately $2-5 \%$ of $\mathrm{AC}$ patients develop end-stage disease, which manifests as severe esophageal dilation, stasis of a large amount of food, or preneoplastic lesions. Patients typically exhibit refractory dysphagia, weight loss, poor nutrition, and frequent reflux. To improve the quality of life of patients and avoid the risk of invasive tumors, esophagectomy may be necessary. ${ }^{[23,56]}$ Approximately $80 \%$ of patients who undergo esophagectomy experience symptom alleviation. However, esophagectomy is a highly invasive treatment modality at risk for multiple surgical complications; postoperative dysphagia caused by anastomotic stricture is the most common complication. As esophagectomy is associated with a mortality rate of up to $5.4 \%$ and a long recovery time, it should only be selected as a treatment alternative after careful consideration. ${ }^{[57]}$

\section{CONCLUSIONS AND OUTLOOK}

AC is an esophageal motor disorder characterized by progressive dysphagia that severely impacts the quality of daily life of patients. Currently, barium esophagography, esophageal manometry (gold standard), endoscopy, and EUS allow for AC to be diagnosed clinically and further classified to provide guidance for subsequent treatment and prognostic assessments, which is of great importance to clinical practice. After a confirmed diagnosis has been made, appropriate treatment regimens can be selected based on the type of AC. Current therapies for the treatment of $\mathrm{AC}$ include conservative pharmacological therapy; minimally invasive endoscopic therapies such as EBTI, EPD, and ESP; and surgical therapies such as myotomy and esophagectomy. Although LHM is the gold standard for the treatment of AC, the recently developed POEM procedure enables the significant reduction of the LES pressure and alleviation of dysphagia symptoms. POEM also provides multiple benefits such as reduced trauma, a short recovery period, an absence of incisions on the body surface, maximum retention of the physiological functions of the esophagus, and reduced postoperative complications. POEM possesses great potential in the development of future minimally invasive treatment modalities for AC. Therefore, conservative drug therapy and Botox injection are generally appropriate only for patients in the early AC stage, frail elderly high-risk patients, and patients who reject other forms of treatment. LHM combined with Dor fundoplication (LHM + Dor) is the first choice for the treatment of AC. Balloon dilation and the emerging POEM technique should be considered for patients seeking less trauma and a faster recovery. Stenting is generally used to support patients at the end of their illness; hence, esophagectomy is used to a lesser extent.
At present, the underlying principle of currently available AC treatment methods is the destruction of the LES for the alleviation of dysphagia symptoms rather than the restoration of normal physiological functions in the LES. In a recent case study, it was reported that a patient with $\mathrm{AC}$ and eosinophilic esophagitis experienced improvement in esophageal motility and disappearance of dysphagia symptoms after treatment with prednisolone. ${ }^{[58]}$ As AC may possibly be an autoimmune-mediated inflammatory response, the use of immunotherapy agents may be considered theoretically. Another possible approach is the restoration of normal functions in the LES through neural stem cell transplantation to achieve the purpose of AC treatment. ${ }^{[23,59]}$

In conclusion, with ongoing improvements in medical technology, a number of effective AC treatment methods can be expected in the future that will benefit patient care and clinical outcomes.

\section{Source of Funding}

National Natural Science Foundation of China (Grant No. 81900601), University innovation team and innovative talent support program of Liaoning Province (Grant No. LR2019073), and Outstanding Scientific Fund of Shengjing Hospital (Grant No. 201702).

\section{Conflict of Interest}

None declared.

\section{REFERENCES}

1. Sadowski DC, Ackah F, Jiang B, Svenson LW. Achalasia incidence, prevalence and survival. A population-based study. Neurogastroenterol Motil 2010; 22: e256-61.

2. Duffield JA, Hamer PW, Heddle R, Holloway RH, Myers JC, Thompson SK. Incidence of Achalasia in South Australia Based on Esophageal Manometry Findings. Clin Gastroenterol Hepatol 2017; 15: 360-365.

3. Samo S, Carlson DA, Gregory DL, Gawel SH, Pandolfino JE, Kahrilas PJ. Incidence and Prevalence of Achalasia in Central Chicago, 20042014, Since the Widespread Use of High-Resolution Manometry. Clin Gastroenterol Hepatol 2017; 15: 366-373.

4. Francisco Schlottmann, Marco G. Patti. Esophageal achalasia: current diagnosis and treatment. Expert Review of Gastroenterology \& Hepatology 2018; 12: 711-721.

5. Vaezi MF, Pandolfino JE, Vela MF. ACG clinical guideline: diagnosis and management of achalasia. Am J Gastroenterol 2013; 108: 1238-49.

6. Furuzawa-Carballeda J, Aguilar-León D, Gamboa-Domínguez A, Valdovinos MA, Nuñez-Álvarez C, Martín-del-Campo LA, et al. Achalasia-An Autoimmune Inflammatory Disease: A Cross-Sectional Study. J Immunol Res 2015; 729217.

7. Kessing BF, Bredenoord AJ, Smout AJ. Erroneous diagnosis of gastroesophageal reflux disease in achalasia. Clin Gastroenterol Hepatol 2011; 9: 1020-4.

8. Jung DH, Park H. Is Gastroesophageal Reflux Disease and Achalasia Coincident or Not? J Neurogastroenterol Motil 2017; 23: 5-8. 
9. Tustumi F, Bernardo WM, da Rocha JRM, Szachnowicz S, Seguro FC, Bianchi ET, et al. Esophageal achalasia: a risk factor for carcinoma. A systematic review and meta-analysis. Dis Esophagus 2017; 30: 1-8.

10. Brücher BL, Stein HJ, Bartels H, Feussner H, Siewert JR. Achalasia and esophageal cancer: incidence, prevalence, and prognosis. World J Surg 2001; 25: 745-9.

11. Fisichella PM, Raz D, Palazzo F, Niponmick I, Patti MG. Clinical, radiological, and manometric profile in 145 patients with untreated achalasia. World J Surg 2008; 32: 1974-9.

12. Rohof WO, Lei A, Boeckxstaens GE. Esophageal stasis on a timed barium esophagogram predicts recurrent symptoms in patients with long-standing achalasia. Am J Gastroenterol 2013; 108: 49-55.

13. Patel DA, Kim HP, Zifodya JS, Vaezi MF. Idiopathic (primary) achalasia: a review. Orphanet J Rare Dis 2015; 10: 89.

14. Richter JE, Boeckxstaens GE. Management of achalasia: surgery or pneumatic dilation. Gut 2011; 60: 869-76.

15. Min M, Peng LH, Yang YS, Hou XH, Guo RB, Wang WF, et al. Characteristics of achalasia subtypes in untreated Chinese patients: a high-resolution manometry Study. J Dig Dis 2012; 13: 504-509.

16. Carlson DA, Pandolfino JE. High-resolution manometry and esophageal pressure topography: filling the gaps of convention Manometry. Gastroenterol Clin North Am 2013; 42: 1-15.

17. Rohof WO, Salvador R, Annese V, Bruley des Varannes S, Chaussade $\mathrm{S}$, Costantini $\mathrm{M}$, et al. Outcomes of treatment for achalasia depend on manometric subtype. Gastroenterology 2013; 144: 681-684.

18. Pandolfino JE, Kwiatek MA, Nealis T, Bulsiewicz W, Post J, Kahrilas PJ. Achalasia: a new clinically relevant classification by high-resolution manometry. Gastroenterology 2008; 135: 1526-33.

19. Rohof WO, Salvador R, Annese V, Bruley des Varannes S, Chaussade $\mathrm{S}$, Costantini $\mathrm{M}$, et al. Outcomes of treatment for achalasia depend on manometric subtype. Gastroenterology 2013; 144: 718-25.

20. Ou YH, Nie XM, Li LF, Wei ZJ, Jiang B. High-resolution manometric subtypes as a predictive factor for the treatment of achalasia: A metaanalysis and systematic review. J Dig Dis 2016; 17: 222-35.

21. Fisichella PM, Raz D, Palazzo F, Niponmick I, Patti MG. Clinical, radiological, and manometric profile in 145 patients with untreated achalasia. World J Surg 2008; 32: 1974-79.

22. El-Takli I, O’Brien P, Paterson WG. Clinical diagnosis of achalasia: how reliable is the barium $x$-ray? Can J Gastroenterol 2006; 20: 335-37.

23. Boeckxstaens GE, Zaninotto G, Richter JE. Achalasia. Lancet 2014; 383 : 83-93.

24. Li HK, Linghu EQ. New endoscopic classification of achalasia for selection of candidates for peroral endoscopic myotomy. World J Gastroenterol 2013; 19: 556-560.

25. Pesenti C, Bories E, Caillol F, Ratone JP, Godat S, Monges G, Poizat F, et al. Characterization of subepithelial lesions of the stomach and esophagus by contrast-enhanced EUS: A retrospective study. Endosc ultrasound 2019; 8: 43-9.

26. Patel S, Jinjuvadia R, Devara A, Naylor PH, Anees M, Jinjuvadia K, et al. Performance characteristics of EUS-FNA biopsy for adrenal lesions: A meta-analysis. Endosc ultrasound 2019; 8: 180-7.

27. Antonini F, Delconte G, Fuccio L, De Nucci G, Fabbri C, Armellini E, et al. EUS-guided tissue sampling with a 20-gauge core biopsy needle for the characterization of gastrointestinal subepithelial lesions: A multicenter study. Endosc ultrasound 2019; 8: 105-10.

28. Ge N, Hu J, Sun S, Linghu E, Jin Z, Li Z. Endoscopic ultrasound-guided pancreatic pseudocyst drainage with lumen-apposing metal stents or plastic double-pigtail stents: A multifactorial analysis. J Transl Intern Med 2017; 5: 213-19.

29. Bhutani MS. EUS-guided botulinum toxin injection into the pyloric sphincter for the treatment of gastroparesis. Endosc ultrasound 2019; 8: 350-1.

30. Kahaleh M, Artifon ELA, Perez-Miranda M, Gaidhane M, Rondon C, Freeman M, et al. EUS-guided drainage: Summary of therapeutic EUS consortium meeting. Endosc ultrasound 2019; 8: 151-60.
31. Larghi A, Rizzatti G, Rimbaş M, Crino SF, Gasbarrini A, Costamagna G. EUS-guided radiofrequency ablation as an alternative to surgery for pancreatic neuroendocrine neoplasms: Who should we treat? Endosc ultrasound 2019; 8: 220-6.

32. Watanabe D, Tanaka S, Ariyoshi R, Abe H, Kawara F, Toyonaga T. Muscle layer thickness affects the peroral endoscopic myotomy procedure complexity. Dis Esophagus 2018; 31: 1-6.

33. Zhong YS, Li L, Zhou PH, Ma LL, Shi Q, Xu MD, et al. The application value of endoscopic ultrasonography examination before esophageal achalasia treated by peroral endoscopic myotomy. Chin J Dig 2012; 11 : 727-730.

34. Minami $\mathrm{H}$, Inoue $\mathrm{H}$, Isomoto $\mathrm{H}$, Urabe $\mathrm{S}$, Nakao K. Clinical application of endoscopic ultrasonography for esophageal achalasia. Dig Endosc 2015; 27 Suppl 1: 11-6.

35. van Hoeij FB, Tack JF, Pandolfino JE, Sternbach JM, Roman S, Smout $\mathrm{AJ}$, et al. Complications of botulinum toxin injections for treatment of esophageal motility disorders. Dis Esophagus 2017; 30: 1-5.

36. Zaninotto G, Bennett C, Boeckxstaens G, Costantini M, Ferguson MK, Pandolfino JE, et al. The 2018 ISDE achalasia guidelines. Dis Esophagus $2018 ; 31$.

37. Ramzan Z, Nassri AB. The role of Botulinum toxin injection in the management of achalasia. Curr Opin Gastroenterol 2013; 29: 468-73.

38. Ruiz Cuesta P, Hervás Molina AJ, Jurado García J, Pleguezuelo Navarro M, García Sánchez V, Casáis Juanena LL, et al. [Pneumatic dilation in the treatment of achalasia]. Gastroenterol Hepatol 2013; 36: 508-12.

39. Aljebreen AM, Samarkandi S, Al-Harbi T, Al-Radhi H, Almadi MA. Efficacy of pneumatic dilatation in Saudi achalasia patients. Saudi J Gastroenterol 2014; 20: 43-7.

40. Vanuytsel T, Lerut T, Coosemans W, Vanbeckevoort D, Blondeau $\mathrm{K}$, Boeckxstaens G, et al. Conservative management of esophageal perforations during pneumatic dilation for idiopathic esophageal achalasia. Clin Gastroenterol Hepatol 2012; 10: 142-49.

41. Schoppmeyer K, Golsong J, Schiefke I, Mössner J, Caca K. Antireflux stents for palliation of malignant esophagocardial stenosis. Dis Esophagus 2007; 20: 89-93.

42. Li YD, Tang GY, Cheng YS, Chen NW, Chen WX, Zhao JG. 13-year follow-up of a prospective comparison of the long-term clinical efficacy of temporary self-expanding metallic stents and pneumatic dilatation for the treatment of achalasia in 120 patients. AJR Am J Roentgenol 2010; 195: 1429-37.

43. Zhao H, Wan XJ, Yang CQ. Comparison of endoscopic balloon dilation with metal stent placement in the treatment of achalasia. Dig Dis 2015; 16: $311-8$

44. Rieder E, Asari R, Paireder M, Lenglinger J, Schoppmann SF. Endoscopic stent suture fixation for prevention of esophageal stent migration during prolonged dilatation for achalasia treatment. Dis Esophagus 2017; 30: $1-6$.

45. Inoue H, Minami H, Kobayashi Y, Sato Y, Kaga M, Suzuki M, et al. Peroral endoscopic myotomy (POEM) for esophageal achalasia. Endoscopy 2010; 42: 265-71.

46. Talukdar R, Inoue H, Nageshwar Reddy D. Efficacy of peroral endoscopic myotomy (POEM) in the treatment of achalasia: a systematic review and meta-analysis. Surg Endosc 2015; 29: 3030-46.

47. Crespin OM, Liu LWC, Parmar A, Jackson TD, Hamid J, Shlomovitz E, et al. Safety and efficacy of POEM for treatment of achalasia: a systematic review of the literature. Surg Endosc 2017; 31: 2187-2201.

48. Stavropoulos SN, Friedel D, Modayil R, Parkman HP. Diagnosis and management of esophageal achalasia. BMJ 2016; 354: i2785.

49. Rösch T, Repici A, Boeckxstaens G. Will Reflux Kill POEM? Endoscopy 2017; 49: 625-628.

50. Wang J, Tan N, Xiao Y, Chen J, Chen B, Ma Z, et al. Safety and efficacy of the modified peroral endoscopic myotomy with shorter myotomy for achalasia patients: a prospective study. Dis Esophagus 2015; 28: 720-7. 
51. Li L, Chai N, Linghu E, Li Z, Du C, Zhang W, et al. Safety and efficacy of using a short tunnel versus a standard tunnel for peroral endoscopic myotomy for Ling type IIc and III achalasia: a retrospective study. Surg Endosc 2019; 33: 1394-1402.

52. Li Y, LingHu E, Ding H, Zhang X, Li M, Xiong Y, et al. Peroral endoscopic myotomy with simultaneous submucosal and muscle dissection for achalasia with severe interlayer adhesions. Gastrointest Endosc 2016; 83: 651-2.

53. Liu BR, Song JT, Omar Jan M. Video of the month. Modified peroral endoscopic myotomy. Am J Gastroenterol 2015; 110: 499.

54. Campos GM, Vittinghoff E, Rabl C, Takata M, Gadenstätter M, Lin F, et al. Endoscopic and surgical treatments for achalasia: a systematic review and meta-analysis. Ann Surg 2009; 249: 45-57.

55. Rebecchi F, Giaccone C, Farinella E, Campaci R, Morino M. Randomized controlled trial of laparoscopic Heller myotomy plus Dor fundoplication versus Nissen fundoplication for achalasia: long-term results. Ann Surg 2008; 248: 1023-30.
56. Pandolfino JE, Gawron AJ. Achalasia: a systematic review. JAMA 2015; 313: 1841-52.

57. Howard JM, Ryan L, Lim KT, Reynolds JV. Oesophagectomy in the management of end-stage achalasia-case reports and a review of the literature. Int J Surg 2011; 9: 204-8.

58. Savarino E, Gemignani L, Zentilin P, De Bortoli N, Malesci A, Mastracci $\mathrm{L}$, et al. Achalasia with dense eosinophilic infi ltrate responds to steroid therapy. Clin Gastroenterol Hepatol 2011; 9: 1104-06.

59. Shaker A, Rubin DC. Stem cells: one step closer to gut repair. Nature 2012; 485: 181-2.

How to cite this article: Zhang B, Wang Y, Liao Y, Zhang J, Wu Y, Xiao T, et al. Advances in the diagnosis and treatment of achalasia of the cardia: a review. J Transl Intern Med 2021; 9: 24-31. 